\title{
Free Interpretation, Quotient Interpretation and Substitution of a Letter with a Term for First Order Languages ${ }^{1}$
}

\author{
Marco B. Caminati ${ }^{2}$ \\ Mathematics Department "G.Castelnuovo" \\ Sapienza University of Rome \\ Piazzale Aldo Moro 5, 00185 Roma, Italy
}

\begin{abstract}
Summary. Fourth of a series of articles laying down the bases for classical first order model theory. This paper supplies a toolkit of constructions to work with languages and interpretations, and results relating them. The free interpretation of a language, having as a universe the set of terms of the language itself, is defined.

The quotient of an interpreteation with respect to an equivalence relation is built, and shown to remain an interpretation when the relation respects it. Both the concepts of quotient and of respecting relation are defined in broadest terms, with respect to objects as general as possible.
\end{abstract}

Along with the trivial symbol substitution generally defined in [11], the more complex substitution of a letter with a term is defined, basing right on the free interpretation just introduced, which is a novel approach, to the author's knowledge. A first important result shown is that the quotient operation commute in some sense with term evaluation and reassignment functors, both introduced in [13] (theorem 3, theorem 15). A second result proved is substitution lemma (theorem 10, corresponding to III.8.3 of [15]). This will be vital for proving satisfiability theorem and correctness of a certain sequent derivation rule in [14]. A third result supplied is that if two given languages coincide on the letters of a given FinSequence, their evaluation of it will also coincide. This too will be instrumental in [14] for proving correctness of another rule. Also, the Depth functor is shown to be invariant with respect to term substitution in a formula.

MML identifier: FOMODEL3, version: $\underline{7.11 .07 \quad 4.160 .1126}$

\footnotetext{
${ }^{1}$ The author wrote this paper as part of his $\mathrm{PhD}$ thesis research.

${ }^{2}$ I would like to thank Marco Pedicini for his encouragement and support.
} 
The notation and terminology used in this paper are introduced in the following articles: [1], [20], [17], [4], [5], [11], [12], [13], [19], [6], [7], [8], [16], [22], [2], [3], [9], [23], [25], [24], [18], [21], and [10].

For simplicity, we adopt the following rules: $X, Y, x$ are sets, $U, U_{1}, U_{2}$ are non empty sets, $u, u_{1}$ are elements of $U, R$ is a binary relation, $f$ is a function, $m, n$ are natural numbers, $m_{1}, n_{1}$ are elements of $\mathbb{N}, S, S_{1}, S_{2}$ are languages, $s$ is an element of $S, l, l_{1}, l_{2}$ are literal elements of $S, a$ is an of-atomic-formula element of $S, r$ is a relational element of $S, w$ is a string of $S, t$ is a termal string of $S, p_{0}$ is a 0 -w.f.f. string of $S, p_{1}, p_{2}$ are w.f.f. strings of $S, I$ is an $(S, U)$-interpreter-like function, and $t_{1}, t_{0}$ are elements of AllTermsOf $S$.

Let us consider $S, s$ and let $V$ be an element of ((AllSymbolsOf $\left.S)^{*} \backslash\{\emptyset\}\right)^{*}$. The functor $s$-compound $V$ yields a string of $S$ and is defined by:

(Def. 1) $s$-compound $V=\langle s\rangle \frown S$-multiCat $(V)$.

Let us consider $S, m_{1}$, let $s$ be a termal element of $S$, and let $V$ be an $|\operatorname{ar} s|$-element element of $S$-termsOfMaxDepth $\left(m_{1}\right)^{*}$. One can verify that $s$-compound $V$ is $m_{1}+1$-termal.

Let us consider $S$, let $s$ be a termal element of $S$, and let $V$ be an $|a r s|$ element element of (AllTermsOf $S)^{*}$. Observe that $s$-compound $V$ is termal.

Let us consider $S$, let $s$ be a relational element of $S$, and let $V$ be an $|\operatorname{ar} s|$ element element of (AllTermsOf $S)^{*}$. One can check that $s$-compound $V$ is 0 w.f.f..

Let us consider $S, s$. The functor $s$-compound yielding a function from $\left((\text { AllSymbolsOf } S)^{*} \backslash\{\emptyset\}\right)^{*}$ into (AllSymbolsOf $\left.S\right)^{*} \backslash\{\emptyset\}$ is defined by:

(Def. 2) For every element $V$ of $\left((\text { AllSymbolsOf } S)^{*} \backslash\{\emptyset\}\right)^{*}$ holds $s$-compound $(V)=$ $s$-compound $V$.

Let us consider $S$ and let $s$ be a termal element of $S$.

Observe that $s$-compound $\left\lceil(\text { AllTermsOf } S)^{|\operatorname{ar} s|}\right.$ is AllTermsOf $S$-valued.

Let us consider $S$ and let $s$ be a relational element of $S$.

Note that $s$-compound $\left\lceil(\text { AllTermsOf } S)^{\mid a r} s \mid\right.$ is AtomicFormulasOf $S$-valued.

Let us consider $S$, let $s$ be an of-atomic-formula element of $S$, and let $X$ be a set. The functor $X$-freeInterpreter $s$ is defined as follows:

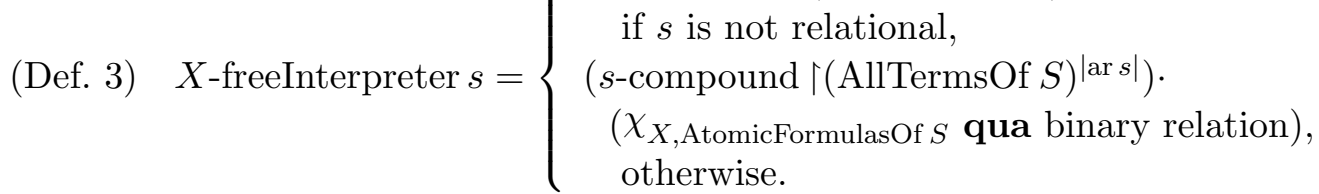

Let us consider $S$, let $s$ be an of-atomic-formula element of $S$, and let $X$ be a set. Then $X$-freeInterpreter $s$ is an interpreter of $s$ and AllTermsOf $S$.

Let us consider $S, X$. The functor $(S, X)$-freeInterpreter yields a function and is defined as follows: 
(Def. 4) $\operatorname{dom}((S, X)$-freeInterpreter $)=$ OwnSymbolsOf $S$ and for every own element $s$ of $S$ holds $(S, X)$-freeInterpreter $(s)=X$-freeInterpreter $s$.

Let us consider $S, X$. Note that $(S, X)$-freeInterpreter is function yielding.

Let us consider $S, X$. Then $(S, X)$-freeInterpreter is an interpreter of $S$ and AllTermsOf $S$.

Let us consider $S, X$. Note that $(S, X)$-freeInterpreter is ( $S$, AllTermsOf $S)$ interpreter-like.

Then $(S, X)$-freeInterpreter is an element of AllTermsOf $S$-InterpretersOf $S$.

Let $X, Y$ be non empty sets, let $R$ be a relation between $X$ and $Y$, and let $n$ be a natural number. The functor $n$-placesOf $R$ yielding a relation between $X^{n}$ and $Y^{n}$ is defined as follows:

(Def. 5) $n$-placesOf $R=\left\{\langle p, q\rangle ; p\right.$ ranges over elements of $X^{n}, q$ ranges over elements of $\left.Y^{n}: \bigwedge_{j: \text { set }}(j \in \operatorname{Seg} n \Rightarrow\langle p(j), q(j)\rangle \in R)\right\}$.

Let $X, Y$ be non empty sets, let $R$ be a total relation between $X$ and $Y$, and let $n$ be a non zero natural number. Observe that $n$-placesOf $R$ is total.

Let $X, Y$ be non empty sets, let $R$ be a total relation between $X$ and $Y$, and let $n$ be a natural number. Observe that $n$-placesOf $R$ is total.

Let $X, Y$ be non empty sets, let $R$ be a relation between $X$ and $Y$, and let $n$ be a zero natural number. One can check that $n$-placesOf $R$ is function-like.

Let $X$ be a non empty set, let $R$ be a binary relation on $X$, and let $n$ be a natural number. The functor $n$-places $O f$ yielding a binary relation on $X^{n}$ is defined by:

(Def. 6) $n$-placesOf $R=n$-placesOf $(R$ qua relation between $X$ and $X)$.

Let $X$ be a non empty set, let $R$ be a binary relation on $X$, and let $n$ be a zero natural number. Then $n$-placesOf $R$ is a binary relation on $X^{n}$ and it can be characterized by the condition:

(Def. 7) $n$-placesOf $R=\{\langle\emptyset, \emptyset\rangle\}$.

Let $X$ be a non empty set, let $R$ be a symmetric total binary relation on $X$, and let us consider $n$. One can check that $n$-placesOf $R$ is total.

Let $X$ be a non empty set, let $R$ be a symmetric total binary relation on $X$, and let us consider $n$. Observe that $n$-placesOf $R$ is symmetric.

Let $X$ be a non empty set, let $R$ be a symmetric total binary relation on $X$, and let us consider $n$. Observe that $n$-placesOf $R$ is symmetric and total.

Let $X$ be a non empty set, let $R$ be a transitive total binary relation on $X$, and let us consider $n$. Observe that $n$-placesOf $R$ is transitive and total.

Let $X$ be a non empty set, let $R$ be an equivalence relation of $X$, and let us consider $n$. Observe that $n$-places $O f$ is total, symmetric, and transitive.

Let $X, Y$ be non empty sets, let $E$ be an equivalence relation of $X$, let $F$ be an equivalence relation of $Y$, and let $R$ be a binary relation. The functor $R$ quotient $(E, F)$ is defined by: 
(Def. 8) $R$ quotient $(E, F)=\{\langle e, f\rangle ; e$ ranges over elements of Classes $E, f$ ranges over elements of Classes $\left.F: \bigvee_{x, y \text { : set }}(x \in e \wedge y \in f \wedge\langle x, y\rangle \in R)\right\}$.

Let $X, Y$ be non empty sets, let $E$ be an equivalence relation of $X$, let $F$ be an equivalence relation of $Y$, and let $R$ be a binary relation. Then $R$ quotient $(E, F)$ is a relation between Classes $E$ and Classes $F$.

Let $E$ be a binary relation, let $F$ be a binary relation, and let $f$ be a function. We say that $f$ is $(E, F)$-respecting if and only if:

(Def. 9) For all sets $x_{1}, x_{2}$ such that $\left\langle x_{1}, x_{2}\right\rangle \in E$ holds $\left\langle f\left(x_{1}\right), f\left(x_{2}\right)\right\rangle \in F$.

Let us consider $S, U$, let $s$ be an of-atomic-formula element of $S$, let $E$ be a binary relation on $U$, and let $f$ be an interpreter of $s$ and $U$. We say that $f$ is $E$-respecting if and only if:

(Def. 10)(i) $\quad f$ is $(|\operatorname{ar} s|$-placesOf $E, E)$-respecting if $s$ is not relational,

(ii) $\quad f$ is $\left(|\operatorname{ar} s|\right.$-placesOf $E$, id $\left._{\text {Boolean }}\right)$-respecting, otherwise.

Let $X, Y$ be non empty sets, let $E$ be an equivalence relation of $X$, and let $F$ be an equivalence relation of $Y$. Observe that there exists a function from $X$ into $Y$ which is $(E, F)$-respecting.

Let us consider $S, U$, let $s$ be an of-atomic-formula element of $S$, and let $E$ be an equivalence relation of $U$. Note that there exists an interpreter of $s$ and $U$ which is $E$-respecting.

Let $X, Y$ be non empty sets, let $E$ be an equivalence relation of $X$, and let $F$ be an equivalence relation of $Y$. One can verify that there exists a function which is $(E, F)$-respecting.

Let $X$ be a non empty set, let $E$ be an equivalence relation of $X$, and let us consider $n$. Then $n$-placesOf $E$ is an equivalence relation of $X^{n}$.

Let $X$ be a non empty set and let $x$ be an element of $\operatorname{SmallestPartition}(X)$. The functor DeTrivial $x$ yielding an element of $X$ is defined as follows:

(Def. 11) $x=\{$ DeTrivial $x\}$.

Let $X$ be a non empty set. The functor peeler $X$ yielding a function from $\{\{*\}: * \in X\}$ into $X$ is defined as follows:

(Def. 12) For every element $x$ of $\{\{*\}: * \in X\}$ holds (peeler $X)(x)=\operatorname{DeTrivial} x$.

Let $X$ be a non empty set and let $E_{1}$ be an equivalence relation of $X$. Note that every element of Classes $E_{1}$ is non empty.

Let $X, Y$ be non empty sets, let $E$ be an equivalence relation of $X$, let $F$ be an equivalence relation of $Y$, and let $f$ be an $(E, F)$-respecting function. One can check that $f$ quotient $(E, F)$ is function-like.

Let $X, Y$ be non empty sets, let $E$ be an equivalence relation of $X$, let $F$ be an equivalence relation of $Y$, and let $R$ be a total relation between $X$ and $Y$. One can check that $R$ quotient $(E, F)$ is total.

Let $X, Y$ be non empty sets, let $E$ be an equivalence relation of $X$, let $F$ be an equivalence relation of $Y$, and let $f$ be an $(E, F)$-respecting function from $X$ 
into $Y$. Then $f$ quotient $(E, F)$ is a function from Classes $E$ into Classes $F$.

Let $X$ be a non empty set and let $E$ be an equivalence relation of $X$. The functor $E$-class yields a function from $X$ into Classes $E$ and is defined by:

(Def. 13) For every element $x$ of $X$ holds $E$-class $(x)=\operatorname{EqClass}(E, x)$.

Let $X$ be a non empty set and let $E$ be an equivalence relation of $X$. Observe that $E$-class is onto.

Let $X, Y$ be non empty sets. Note that there exists a relation between $X$ and $Y$ which is onto.

Let $Y$ be a non empty set. Observe that there exists a $Y$-valued binary relation which is onto.

Let $Y$ be a non empty set and let $R$ be a $Y$-valued binary relation. Note that $R^{\smile}$ is $Y$-defined.

Let $Y$ be a non empty set and let $R$ be an onto $Y$-valued binary relation. Note that $R^{\smile}$ is total.

Let $X, Y$ be non empty sets and let $R$ be an onto relation between $X$ and $Y$. One can check that $R^{\smile}$ is total.

Let $Y$ be a non empty set and let $R$ be an onto $Y$-valued binary relation. Note that $R^{\smile}$ is total.

Let us consider $U, n$ and let $E$ be an equivalence relation of $U$. The functor $n$-tuple2Class $E$ yields a relation between $(\text { Classes } E)^{n}$ and Classes $(n$-placesOf $E)$ and is defined as follows:

(Def. 14) $n$-tuple2Class $E=(n$-placesOf( $E$-class qua relation between $U$ and Classes $\left.E)^{\smile}\right) \cdot(n$-placesOf $E)$-class .

Let us consider $U, n$ and let $E$ be an equivalence relation of $U$. Observe that $n$-tuple2Class $E$ is function-like.

Let us consider $U, n$ and let $E$ be an equivalence relation of $U$. Note that $n$-tuple2Class $E$ is total.

Let us consider $U, n$ and let $E$ be an equivalence relation of $U$. Then $n$-tuple2Class $E$ is a function from (Classes $E)^{n}$ into Classes (n-placesOf $E$ ).

Let us consider $S, U$, let $s$ be an of-atomic-formula element of $S$, let $E$ be an equivalence relation of $U$, and let $f$ be an interpreter of $s$ and $U$. The functor $f$ quotient $E$ is defined by:

(Def. 15) $f$ quotient $E=\left\{\begin{array}{l}(|\operatorname{ar} s| \text {-tuple2Class } E) . \\ (f \text { quotient }(|\operatorname{ar} s| \text {-placesOf } E, E)) \\ \text { if } s \text { is not relational, } \\ (|\operatorname{ar} s| \text {-tuple2Class } E) \cdot \\ \left(f \text { quotient }\left(|\operatorname{ar} s| \text {-placesOf } E, \text { id }_{\text {Boolean }}\right)\right) \\ \text { peeler Boolean, otherwise. }\end{array}\right.$

Let us consider $S, U$, let $s$ be an of-atomic-formula element of $S$, let $E$ be an equivalence relation of $U$, and let $f$ be an $E$-respecting interpreter of $s$ and $U$. Then $f$ quotient $E$ is an interpreter of $s$ and Classes $E$. 
The following proposition is true

(1) Let $X$ be a non empty set, $E$ be an equivalence relation of $X$, and $C_{1}$, $C_{2}$ be elements of Classes $E$. If $C_{1}$ meets $C_{2}$, then $C_{1}=C_{2}$.

Let us consider $S$. Observe that every element of OwnSymbolsOf $S$ is own and every element of OwnSymbolsOf $S$ is of-atomic-formula.

Let us consider $S, U$, let $o$ be a non relational of-atomic-formula element of $S$, and let $E$ be a binary relation on $U$. One can check that every interpreter of $o$ and $U$ which is $E$-respecting is also ( $|\operatorname{ar} o|$-placesOf $E, E$ )-respecting.

Let us consider $S, U$, let $r$ be a relational element of $S$, and let $E$ be a binary relation on $U$. Observe that every interpreter of $r$ and $U$ which is $E$-respecting is also ( $|\operatorname{ar} r|$-placesOf $\left.E, \mathrm{id}_{\text {Boolean }}\right)$-respecting.

Let us consider $n$, let $U_{1}, U_{2}$ be non empty sets, and let $f$ be a function-like relation between $U_{1}$ and $U_{2}$. Note that $n$-places of $f$ is function-like.

Let us consider $U_{1}, U_{2}$, let $n$ be a zero natural number, and let $R$ be a relation between $U_{1}$ and $U_{2}$. Note that $(n$-placesOf $R)-\mathrm{id}_{\{\emptyset\}}$ is empty.

Let us consider $X$ and let $Y$ be a functional set. Observe that $X \cap Y$ is functional.

We now state the proposition

(2) For every element $V$ of (AllTermsOf $S)^{*}$ there exists an element $m_{1}$ of $\mathbb{N}$ such that $V$ is an element of $S$-termsOfMaxDepth $\left(m_{1}\right)^{*}$.

Let us consider $S, U$, let $E$ be an equivalence relation of $U$, and let $I$ be an $(S, U)$-interpreter-like function. We say that $I$ is $E$-respecting if and only if:

(Def. 16) For every own element $s$ of $S$ holds $I(s)$ qua interpreter of $s$ and $U$ is E-respecting.

Let us consider $S, U$, let $E$ be an equivalence relation of $U$, and let $I$ be an $(S, U)$-interpreter-like function. The functor $I$ quotient $E$ yielding a function is defined as follows:

(Def. 17) $\operatorname{dom}(I$ quotient $E)=$ OwnSymbolsOf $S$ and for every element $o$ of OwnSymbolsOf $S$ holds $(I$ quotient $E)(o)=I(o)$ quotient $E$.

Let us consider $S, U$, let $E$ be an equivalence relation of $U$, and let $I$ be an $(S, U)$-interpreter-like function. Then $I$ quotient $E$ can be characterized by the condition:

(Def. 18) $\operatorname{dom}(I$ quotient $E)=$ OwnSymbolsOf $S$ and for every own element $o$ of $S$ holds $(I$ quotient $E)(o)=I(o)$ quotient $E$.

Let us consider $S, U$, let $I$ be an $(S, U)$-interpreter-like function, and let $E$ be an equivalence relation of $U$. Note that $I$ quotient $E$ is OwnSymbolsOf $S$ defined.

Let us consider $S, U$ and let $E$ be an equivalence relation of $U$. Note that there exists an element of $U$-InterpretersOf $S$ which is $E$-respecting. 
Let us consider $S, U$ and let $E$ be an equivalence relation of $U$. Observe that there exists an $(S, U)$-interpreter-like function which is $E$-respecting.

Let us consider $S, U$, let $E$ be an equivalence relation of $U$, let $o$ be an own element of $S$, and let $I$ be an $E$-respecting $(S, U)$-interpreter-like function. One can check that $I(o)$ is $E$-respecting.

Let us consider $S, U$, let $E$ be an equivalence relation of $U$, and let $I$ be an $E$-respecting $(S, U)$-interpreter-like function. Observe that $I$ quotient $E$ is $(S$, Classes $E)$-interpreter-like.

Let us consider $S, U$, let $E$ be an equivalence relation of $U$, and let $I$ be an $E$-respecting $(S, U)$-interpreter-like function. Then $I$ quotient $E$ is an element of Classes E-InterpretersOf $S$.

The following propositions are true:

(3) Let $E$ be an equivalence relation of $U$ and $I$ be an $E$-respecting $(S, U)$ interpreter-like function.

Then $(I$ quotient $E)$-TermEval $=E$-class $\cdot I$-TermEval .

(4) $(S, X)$-freeInterpreter-TermEval $=\operatorname{id}_{\text {AllTermsOf } S \text {. }}$

(5) Let $R$ be an equivalence relation of $U_{1}, p_{2}$ be a 0 -w.f.f. string of $S$, and $i$ be an $R$-respecting $\left(S, U_{1}\right)$-interpreter-like function. If $S$-firstChar $\left(p_{2}\right) \neq$ TheEqSymbOf $S$, then $\left(i\right.$ quotient $R$ )-AtomicEval $p_{2}=i$-AtomicEval $p_{2}$.

Let us consider $S, x, s, w$. Then $(x, s)$-SymbolSubstIn $w$ is a string of $S$.

Let us consider $S, l_{1}, l_{2}, m$ and let $t$ be an $m$-termal string of $S$. Note that $\left(l_{1}, l_{2}\right)$-SymbolSubstIn $t$ is $m$-termal.

Let us consider $S, t, l_{1}, l_{2}$. One can check that $\left(l_{1}, l_{2}\right)$-SymbolSubstIn $t$ is termal.

Let us consider $S, l_{1}, l_{2}$ and let $p_{2}$ be a 0 -w.f.f. string of $S$. One can check that $\left(l_{1}, l_{2}\right)$-SymbolSubstIn $p_{2}$ is 0 -w.f.f..

Let us consider $S$, let $m_{0}$ be a zero number, and let $p_{2}$ be an $m_{0}$-w.f.f. string of $S$. One can verify that Depth $p_{2}$ is zero.

Let us consider $S, m, w$. Then $w$ null $m$ is a string of $S$.

Let us consider $S, p_{2}, m$. Note that $p_{2}$ null $m$ is Depth $p_{2}+m$-w.f.f..

Let us consider $S, m$ and let $p_{2}$ be an $m$-w.f.f. string of $S$. Note that $m-$ Depth $p_{2}$ is non negative.

Let us consider $S, l_{1}, l_{2}, m$ and let $p_{2}$ be an $m$-w.f.f. string of $S$. Observe that $\left(l_{1}, l_{2}\right)$-SymbolSubstIn $p_{2}$ is $m$-w.f.f..

Let us consider $S, l_{1}, l_{2}, p_{2}$. One can verify that $\left(l_{1}, l_{2}\right)$-SymbolSubstIn $p_{2}$ is w.f.f.. Observe that $\operatorname{Depth}\left(\left(l_{1}, l_{2}\right)\right.$-SymbolSubstIn $\left.p_{2}\right)-\operatorname{Depth} p_{2}$ is empty.

The following proposition is true

(6) Let $T$ be an $|\operatorname{ar} a|$-element element of (AllTermsOf $S)^{*}$. Then

(i) if $a$ is not relational, then $(X$-freeInterpreter $a)(T)=a$-compound $T$, and

(ii) if $a$ is relational, then $(X$-freeInterpreter $a)(T)=$ 
$\chi_{X, \text { AtomicFormulasOf } S}(a$-compound $T)$.

Let $S$ be a language. One can verify that there exists a string of $S$ which is termal and there exists a string of $S$ which is 0 -w.f.f..

One can prove the following proposition

(7) $\left(I\right.$-TermEval $\cdot\left(\left(l, t_{0}\right)\right.$ ReassignIn $(S, X)$-freeInterpreter, $\left.t_{0}\right)$-TermEval $\left.(n)\right) \uparrow$ $S$-termsOfMaxDepth $(n)=$ $\left(\left(l, I\right.\right.$-TermEval $\left.\left(t_{0}\right)\right)$ ReassignIn $I, I$-TermEval $\left.\left(t_{0}\right)\right)$-TermEval $(n) \uparrow$ $S$-termsOfMaxDepth $(n)$.

Let us consider $S, l, t_{1}, p_{0}$. The functor $\left(l, t_{1}\right)$ AtomicSubst $p_{0}$ yielding a finite sequence is defined by:

(Def. 19) $\left(l, t_{1}\right)$ AtomicSubst $p_{0}=\left\langle S\right.$-firstChar $\left.\left(p_{0}\right)\right\rangle-S$-multiCat $\left(\left(\left(l, t_{1}\right)\right.\right.$ ReassignIn $(S, \emptyset)$-freeInterpreter)-TermEval $\cdot$ SubTerms $\left.p_{0}\right)$.

Let us consider $S, l, t_{1}, p_{0}$. Then $\left(l, t_{1}\right)$ AtomicSubst $p_{0}$ is a string of $S$.

Let us consider $S, l, t_{1}, p_{0}$. Observe that $\left(l, t_{1}\right)$ AtomicSubst $p_{0}$ is 0 -w.f.f..

We now state the proposition

(8) $I$-AtomicEval $\left(\left(l, t_{1}\right)\right.$ AtomicSubst $\left.p_{0}\right)=$ $\left(\left(l, I\right.\right.$-TermEval $\left.\left(t_{1}\right)\right)$ ReassignIn $\left.I\right)$-AtomicEval $p_{0}$.

Let us consider $S, l_{1}, l_{2}, m$. One can check that $\left(l_{1} \operatorname{SubstWith} l_{2}\right) \uparrow$ $S$-termsOfMaxDepth $(m)$ is $S$-termsOfMaxDepth $(m)$-valued.

Note that $\left(l_{1}\right.$ SubstWith $\left.l_{2}\right)\lceil$ AllTermsOf $S$ is AllTermsOf $S$-valued.

One can prove the following proposition

(9) If $l_{2} \notin \operatorname{rng} p_{1}$, then for every element $I$ of $U$-InterpretersOf $S$ holds $\left(\left(l_{1}, u_{1}\right)\right.$ ReassignIn $\left.I\right)$-TruthEval $p_{1}=$ $\left(\left(l_{2}, u_{1}\right)\right.$ ReassignIn $\left.I\right)-\operatorname{TruthEval}\left(\left(l_{1}, l_{2}\right)\right.$-SymbolSubstIn $\left.p_{1}\right)$.

Let us consider $S$, let us consider $l, t, n$, let $f$ be a finite sequence-yielding function, and let us consider $p_{2}$. The functor $(l, t, n, f)$ Subst $2 p_{2}$ yielding a finite sequence is defined by:

(Def. 20) $\quad(l, t, n, f)$ Subst2 $p_{2}=\left\{\begin{array}{l}\left.\left.\text { head } p_{2} \cup\{l\}\right)\right\rangle^{\frown f} f\left(\left(S \text {-firstChar }\left(p_{2}\right),\right.\right. \\ \text { the element of LettersOf } S \backslash(\operatorname{rng} t \cup \operatorname{rng}\end{array}\right.$ head $\left.\left.p_{2} \cup\{l\}\right)\right)$-SymbolSubstIn head $p_{2}$ ), if Depth $p_{2}=n+1$ and $p_{2}$ is exal and $S$-firstChar $\left(p_{2}\right) \neq l$,

$f\left(p_{2}\right)$, otherwise.

Let us consider $S$. One can verify that every element of (AllFormulas $O f S$ AllFormulasOf $S$ is finite sequence-yielding.

Let us consider $l, t, n$, let $f$ be an element of (AllFormulasOf $S$ ) AllFormulasOf $S$, and let us consider $p_{2}$. Then $(l, t, n, f) \operatorname{Subst} 2 p_{2}$ is a w.f.f. string of $S$. Let $f$ be 
an element of (AllFormulasOf $S)^{\text {AllFormulasOf } S}$, and let us consider $p_{2}$. Observe that $(l, t, n, f) \operatorname{Subst} 2 p_{2}$ is w.f.f..

Let us consider $n_{1}$, let $f$ be an element of (AllFormulasOf $S$ ) AllFormulasOf $S$, and let us consider $p_{2}$. Then $\left(l, t, n_{1}, f\right)$ Subst $2 p_{2}$ is an element of AllFormulasOf $S$.

Let us consider $S, l, t, n$ and let $f$ be an element of

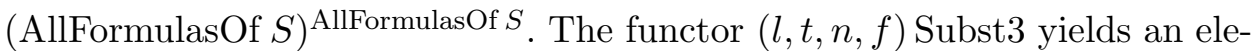
ment of (AllFormulasOf $S$ ) AllFormulasOf $S$ and is defined as follows:

(Def. 21) For every $p_{2}$ holds $(l, t, n, f) \operatorname{Subst} 3\left(p_{2}\right)=(l, t, n, f) \operatorname{Subst} 2 p_{2}$.

Let us consider $S, l, t$ and let $f$ be an element of

(AllFormulasOf $S$ ) AllFormulasOf $S$. The functor $(l, t)$ Subst $4 f$ yields a function from $\mathbb{N}$ into

(AllFormulasOf $S$ ) AllFormulasOf $S$ and is defined by:

(Def. 22) $((l, t)$ Subst $4 f)(0)=f$ and for every $m$ holds $((l, t)$ Subst $4 f)(m+1)=$ $(l, t, m,((l, t)$ Subst4 $f)(m))$ Subst3 .

Let us consider $S, l, t$. The functor $l$ AtomicSubst $t$ yields a function from AtomicFormulas of $S$ into AtomicFormulasOf $S$ and is defined by:

(Def. 23) For all $p_{0}, t_{1}$ such that $t_{1}=t$ holds $(l$ AtomicSubst $t)\left(p_{0}\right)=$ $\left(l, t_{1}\right)$ AtomicSubst $p_{0}$.

Let us consider $S, l, t$. The functor $l$ Subst1 $t$ yielding a function is defined as follows:

(Def. 24) $l$ Subst1 $t=\mathrm{id}_{\text {AllFormulasOf } S}+\cdot(l$ AtomicSubst $t)$.

Let us consider $S, l, t$. Then $l$ Subst $1 t$ is an element of

$\left((\text { AllSymbolsOf } S)^{*}\right)^{\text {AllFormulasOf } S}$. Then $l$ Subst1 $t$ is an element of

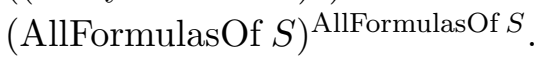

Let us consider $S, l, t, p_{2}$. The functor $(l, t)$ SubstIn $p_{2}$ yielding a w.f.f. string of $S$ is defined as follows:

(Def. 25) $\quad(l, t) \operatorname{SubstIn} p_{2}=((l, t) \operatorname{Subst} 4(l \operatorname{Subst} 1 t))\left(\operatorname{Depth} p_{2}\right)\left(p_{2}\right)$.

Let us consider $S, l, t, p_{2}$. Note that $(l, t)$ SubstIn $p_{2}$ is w.f.f..

One can prove the following proposition

(10) $\operatorname{Depth}\left(\left(l, t_{1}\right) \operatorname{SubstIn} p_{1}\right)=\operatorname{Depth} p_{1}$ and for every element $I$ of $U$-InterpretersOf $S$ holds $I$-TruthEval $\left(\left(l, t_{1}\right)\right.$ SubstIn $\left.p_{1}\right)=$ $\left(\left(l, I\right.\right.$-TermEval $\left.\left(t_{1}\right)\right)$ ReassignIn $\left.I\right)$-TruthEval $p_{1}$.

Let us consider $m, S, l, t$ and let $p_{2}$ be an $m$-w.f.f. string of $S$. Observe that $(l, t) \operatorname{SubstIn} p_{2}$ is $m$-w.f.f..

The following propositions are true:

(11) Let $I_{1}$ be an element of $U$-InterpretersOf $S_{1}$ and $I_{2}$ be an element of $U$-InterpretersOf $S_{2}$. Suppose $I_{1} \uparrow X=I_{2}\left\lceil X\right.$ and (the adicity of $\left.S_{1}\right) \uparrow X=$ (the adicity of $\left.S_{2}\right) \uparrow X$. Then $I_{1}$-TermEval $\uparrow X^{*}=I_{2}$-TermEval $\uparrow X^{*}$. 
(12) Suppose TheNorSymbOf $S_{1}=$ TheNorSymbOf $S_{2}$ and TheEqSymbOf $S_{1}=$ TheEqSymbOf $S_{2}$ and (the adicity of $\left.S_{1}\right)\left\lceil\right.$ OwnSymbolsOf $S_{1}=$ (the adicity of $\left.S_{2}\right)\left\lceil\right.$ OwnSymbolsOf $S_{1}$. Let $I_{1}$ be an element of $U$-InterpretersOf $S_{1}$, $I_{2}$ be an element of $U$-InterpretersOf $S_{2}$, and $p_{4}$ be a w.f.f. string of $S_{1}$. Suppose $\left.I_{1}\right\rceil$ OwnSymbolsOf $S_{1}=I_{2} \uparrow$ OwnSymbolsOf $S_{1}$. Then there exists a w.f.f. string $p_{3}$ of $S_{2}$ such that $p_{3}=p_{4}$ and $I_{2}$-TruthEval $p_{3}=$ $I_{1}$-TruthEval $p_{4}$.

(13) For all elements $I_{1}, I_{2}$ of $U$-InterpretersOf $S$ such that $I_{1} \uparrow\left(\operatorname{rng} p_{2} \cap\right.$ OwnSymbolsOf $S)=I_{2} \uparrow\left(\operatorname{rng} p_{2} \cap\right.$ OwnSymbolsOf $\left.S\right)$ holds $I_{1}$-TruthEval $p_{2}=$ $I_{2}$-TruthEval $p_{2}$.

(14) For every element $I$ of $U$-InterpretersOf $S$ such that $l$ is $X$-absent and $X$ is $I$-satisfied holds $X$ is $(l, u)$ ReassignIn $I$-satisfied.

(15) For every equivalence relation $E$ of $U$ and for every $E$-respecting element $i$ of $U$-Interpreters Of $S$ holds $(l, E$-class $(u))$ ReassignIn $(i$ quotient $E)=$ $((l, u)$ ReassignIn $i)$ quotient $E$.

\section{REFERENCES}

[1] Grzegorz Bancerek. Cardinal numbers. Formalized Mathematics, 1(2):377-382, 1990.

[2] Grzegorz Bancerek. The fundamental properties of natural numbers. Formalized Mathematics, 1(1):41-46, 1990.

[3] Grzegorz Bancerek. The ordinal numbers. Formalized Mathematics, 1(1):91-96, 1990.

[4] Grzegorz Bancerek and Krzysztof Hryniewiecki. Segments of natural numbers and finite sequences. Formalized Mathematics, 1(1):107-114, 1990.

[5] Czesław Byliński. Finite sequences and tuples of elements of a non-empty sets. Formalized Mathematics, 1(3):529-536, 1990.

[6] Czesław Byliński. Functions and their basic properties. Formalized Mathematics, 1(1):5565, 1990.

[7] Czesław Byliński. Functions from a set to a set. Formalized Mathematics, 1(1):153-164, 1990.

[8] Czesław Byliński. The modification of a function by a function and the iteration of the composition of a function. Formalized Mathematics, 1(3):521-527, 1990.

[9] Czesław Byliński. Partial functions. Formalized Mathematics, 1(2):357-367, 1990.

[10] Czesław Byliński. Some basic properties of sets. Formalized Mathematics, 1(1):47-53, 1990.

[11] Marco B. Caminati. Preliminaries to classical first order model theory. Formalized Mathematics, 19(3):155-167, 2011, doi: 10.2478/v10037-011-0025-2.

[12] Marco B. Caminati. Definition of first order language with arbitrary alphabet. Syntax of terms, atomic formulas and their subterms. Formalized Mathematics, 19(3):169-178, 2011, doi: 10.2478/v10037-011-0026-1.

[13] Marco B. Caminati. First order languages: Further syntax and semantics. Formalized Mathematics, 19(3):179-192, 2011, doi: 10.2478/v10037-011-0027-0.

[14] Marco B. Caminati. Sequent calculus, derivability, provability. Gödel's completeness theorem. Formalized Mathematics, 19(3):205-222, 2011, doi: 10.2478/v10037-011-0029-y.

[15] H.D. Ebbinghaus, J. Flum, and W. Thomas. Mathematical logic. Springer, 1994.

[16] Rafał Kwiatek and Grzegorz Zwara. The divisibility of integers and integer relative primes. Formalized Mathematics, 1(5):829-832, 1990.

[17] Konrad Raczkowski and Paweł Sadowski. Equivalence relations and classes of abstraction. Formalized Mathematics, 1(3):441-444, 1990.

[18] Krzysztof Retel. Properties of first and second order cutting of binary relations. Formalized Mathematics, 13(3):361-365, 2005. 
[19] Andrzej Trybulec. Binary operations applied to functions. Formalized Mathematics, 1(2):329-334, 1990

[20] Andrzej Trybulec. Domains and their Cartesian products. Formalized Mathematics, 1(1):115-122, 1990.

[21] Zinaida Trybulec. Properties of subsets. Formalized Mathematics, 1(1):67-71, 1990.

[22] Edmund Woronowicz. Many-argument relations. Formalized Mathematics, 1(4):733-737, 1990.

[23] Edmund Woronowicz. Relations and their basic properties. Formalized Mathematics, 1(1):73-83, 1990.

[24] Edmund Woronowicz. Relations defined on sets. Formalized Mathematics, 1(1):181-186, 1990.

[25] Edmund Woronowicz and Anna Zalewska. Properties of binary relations. Formalized Mathematics, 1(1):85-89, 1990.

Received December 29, 2010 